\title{
Das DFG-Graduiertenkolleg GRK 2297 „Mathematische Komplexitätsreduktion“
}

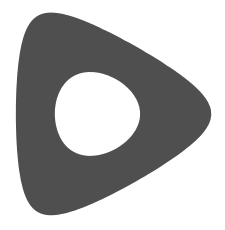

Das DFG-Graduiertenkolleg GRK 2297 zum Thema „Mathematische Komplexitätsreduktion“ (MATHCoRE, www.mathcore.ovgu.de) wurde zum April 2017 an der Otto-von-Guericke Universität Magdeburg eingerichtet.

Die Förderung durch die Deutsche Forschungsgemeinschaft umfasst 15 Promotionsstellen und eine Postdoktorandinnenstelle. Sprecher des Graduiertenkollegs ist Prof. Dr. Sebastian Sager.

Übergreifendes thematisches Ziel des Graduiertenkollegs ist die Entwicklung und die Untersuchung von Ansätzen zur Reduktion von Komplexität auf drei Ebenen: der mathematischen Modellierung realer Probleme, der strukturellen Beschreibung mathematischer Objekte und der algorithmischen Lösung mathematischer Aufgabenstellungen. Dabei decken die beteiligten Arbeitsgruppen ein breites Spektrum von Disziplinen in den Bereichen Numerik, Stochastik, Optimierung, Systemtheorie, Kombinatorik, Algebra und Geometrie ab.
Polynome und semialgebraische Mengen, insbesondere auch konvexe Mengen wie Spektraeder und Polytope, spielen wichtige Rollen in den Forschungsarbeiten im Graduiertenkolleg. Das spiegelt sich im (links gezeigten) MathCoRe-Logo wieder, welches aus Schnittkurven des Elliptops

$$
\left\{\left(x_{1}, x_{2}, x_{2}\right) \in \mathbb{R}^{3}:\left(\begin{array}{ccc}
1 & x_{1} & x_{2} \\
x_{1} & 1 & x_{3} \\
x_{2} & x_{3} & 1
\end{array}\right) \text { ist positiv semidefinit }\right\}
$$

mit zwei zueinander parallelen Ebenen gebildet ist.

Frank Röttger hat in diesem Frühjahr die Reihe der im Graduiertenkolleg fertig gestellten Promotionen eröffnet. Im folgenden, gemeinsam mit seinen beiden Betreuern Rainer Schwabe und Thomas Kahle verfassten Beitrag zur Geometrie optimaler Versuchspläne gibt er einen Einblick in das zwei klassische mathematische Disziplinen verbindende Gebiet seiner Dissertation, die Algebraische Statistik.

\section{Geometrie optimaler Versuchspläne}

\author{
Thomas Kahle, Frank Röttger und Rainer Schwabe
}

\begin{abstract}
Fast jede Entscheidung ist mit Unsicherheiten behaftet und die Wissenschaft soll uns ermöglichen, Risiken zu bewerten und optimal zu handeln. Im Graduiertenkolleg „Mathematische Komplexitätsreduktion“ wird auch die Theorie der statistischen Versuchsplanung untersucht, die helfen soll, Experimente und

Datengewinnung möglichst effizient und informativ zu gestalten.
\end{abstract}

Komplexität soll sichtbar gemacht und dann reduziert werden, wodurch wir Risikokompetenz erlangen und auch unter Unsicherheiten methodisch vorgehen können. Wir wollen die Möglichkeiten und Prinzipien zunächst in einem kleinen Beispiel einführen.

Es sollen acht Objekte $O_{1}, \ldots, O_{8}$ mit unbekannten Gewichten $g_{1}, \ldots, g_{8}$ mittels einer Balkenwaage vermessen werden. Dazu stehen uns kalibrierte Gegengewichte zur Verfü- gung. Die Gegengewichte und Objekte können dabei auf beiden Seiten platziert werden. Die Balkenwaage funktioniert bekanntlich so, dass man durch Ausgleich beider Seiten feststellen kann, dass auf beiden Seiten ungefähr die gleiche Kraft wirkt, die Objekte in den beiden Schalen also ungefähr das gleiche Gewicht haben. Bei jeder Wägung machen wir einen kleinen Fehler, weil wir die Waage nicht perfekt austarieren können. Diese Fehler sind zufällig. Wir 